\title{
A search for Low Surface Brightness galaxies in the near-infrared
}

\section{Selection of the sample ${ }^{\star}$}

\author{
D. Monnier Ragaigne ${ }^{1}$, W. van Driel ${ }^{1}$, S. E. Schneider ${ }^{2}$, T. H. Jarrett ${ }^{3}$, and C. Balkowski ${ }^{1}$ \\ 1 Observatoire de Paris, GEPI, CNRS UMR 8111 and Université Paris 7, 5 place Jules Janssen, 92195 Meudon Cedex, France \\ e-mail: delphine.ragaigne@obspm.fr; wim.vandriel@obspm.fr; chantal.balkowski@obspm.fr \\ 2 University of Massachusetts, Astronomy Program, 536 LGRC, Amherst, MA 01003, USA \\ e-mail: schneide@messier . astro.umass . edu \\ 3 IPAC, Caltech, MS 100-22, 770 South Wilson Ave., Pasadena, CA 91125, USA \\ e-mail: jarrett@ipac.caltech.edu
}

Received 14 February 2003 / Accepted 16 April 2003

\begin{abstract}
A sample of about 3800 Low Surface Brightness (LSB) galaxies was selected using the all-sky near-infrared $(J, H$ and $K_{\mathrm{s}}$-band) 2MASS survey. The selected objects have a mean central surface brightness within a $5^{\prime \prime}$ radius around their centre fainter than $18 \mathrm{mag} \mathrm{arcsec}^{-2}$ in the $K_{\mathrm{s}}$ band, making them the lowest surface brightness galaxies detected by 2MASS. A description is given of the relevant properties of the 2MASS survey and the LSB galaxy selection procedure, as well as of basic photometric properties of the selected objects. The latter properties are compared to those of other samples of galaxies, of both LSBs and "classical" high surface brightness (HSB) objects, which were selected in the optical. The 2MASS LSBs have a $B_{\mathrm{T}_{\mathrm{c}}}-K_{\mathrm{T}}$ colour which is on average 0.9 mag bluer than that of HSBs from the NGC. The 2MASS sample does not appear to contain a significant population of red objects.
\end{abstract}

Key words. galaxies: fundamental parameters - galaxies: general - galaxies: photometry - infrared: galaxies

\section{Introduction}

Observational bias in the selection of galaxies dates back to the visual searches by Messier and Herschel, as galaxies are diffuse objects selected in the presence of a contaminating signal: the brightness of the night sky. The night sky acts as a filter, which, when convolved with the true population of galaxies gives the population of galaxies we observe and which hampers the detection of low surface brightness objects. Based on this concern, the result of the study of Freeman (1970), which indicated a remarkably narrow range of central disc surface brightness values of spiral galaxies in the $B$-band $\left(21.7 \pm 0.3 \mathrm{mag} \operatorname{arcsec}^{-2}\right)$, was questioned by Disney (1976).

In the past few decades, observations of the local universe have shown the existence of galaxies well below the surface brightness of the average previously catalogued galaxies, and often even well below the surface brightness of the night sky, which are referred to as Low Surface Brightness (LSB) galaxies.

Send offprint requests to: $\mathrm{W}$. van Driel, e-mail: wim.vandriel@obspm.fr

* All tables and Figs. 2a-c are only available in electronic form at http://www.edpsciences.org
At present, the LSBs constitute the least well known fraction of galaxies: their number density and physical properties (like luminosity, colours, dynamics) are still quite uncertain. This is, per definition, mainly due to the fundamental difficulty in identifying them in imaging surveys and in measuring their properties. It is far from obvious that all existing classes and types of LSBs have been probed, given the limitations in sensitivity of the actual detectors and the biases in the selection criteria of the published samples.

In order to further investigate the often baffling properties of the LSB class of galaxies we selected a large sample of them from the 2MASS database, accessing the near-infrared, a wavelength domain that has been much less fully explored than the optical in the study of LSBs.

To date, the following studies were published on the nearinfrared properties of LSB galaxies: Knezek \& Wroten (1994) observed a small sample of mainly massive galaxies in $J H K$, Bergvall et al. (1999) observed 14 blue LSBs in JHK and compared their results to optical photometry, Bell et al. (2000) observed $26 \mathrm{LSBs}$ in $J H K$ and a subset of these in $B V R$, de Jong (1996) included a few LSBs among the spiral galaxies he observed in $H$ and $K$, and Galaz et al. (2002) observed 88 LSBs in $J$ and $K_{\mathrm{s}}$. 
Although there is no unambiguous definition of LSB galaxy, those in common use are based on (1) the mean blue surface brightness within the $25 \mathrm{mag} \operatorname{arcsec}^{-2}$ isophote, (2) the mean surface brightness within the half-light radius, or (3) the extrapolated central surface brightness of the disc component alone, after carrying out a disc-bulge decomposition.

For 2MASS galaxies, we used the mean $K_{\mathrm{s}}$-band magnitude within a fixed aperture to identify a sample of galaxies with relatively low infrared surface brightness. We selected galaxies in which the central surface brightness within a $5^{\prime \prime}$ radius circular aperture was fainter than $18 \mathrm{mag} \mathrm{arcsec}^{-2}$ in the $K_{\mathrm{s}}$-band. This criterion selects a relatively small fraction $(<10 \%)$ of large angular diameter sources detected by 2MASS, increasing to about $40 \%$ for the small angular diameter sources detected in the survey.

If 2MASS detections have $B-K=4.0$ colors typical of early-type galaxies (Jarrett et al. 2003; also see Sect. 4.4), as might be anticipated for a sample of infrared-selected sources, this criterion would correspond roughly to the disc-component definition that LSB galaxies are objects with a blue central surface brightness $\mu_{B_{0}}>22.0 \mathrm{mag} \operatorname{arcsec}^{-2}$ (i.e., more than $1 \sigma$ fainter than the $21.7 \pm 0.3 \mathrm{mag} \operatorname{arcsec}^{-2}$ measured by Freeman 1970). Our sample galaxies can have an even lower disc surface brightness if the bulge component is significant, but since we average over a fixed angular aperture, on the other hand we may also include some higher central surface brightness sources that are more distant so that the aperture includes more of the disc. In this paper, we examine the properties of our sample based on 2MASS and LEDA data and compare it to optically-selected galaxy samples.

LSBs have remarkable properties which distinguish them from "classical" High Surface Brightness (HSB) spirals, notably:

- LSBs seem to constitute at least $50 \%$ of the total galaxy population in number in the local Universe, which may have strong implications for the faint end slope of the galaxy luminosity function, on the baryonic matter density and especially on galaxy formation scenarios (O'Neil \& Bothun 2000).

- LSBs discs are among the less evolved objects in the local universe since they have a very low star formation rate (van der Hulst et al. 1993; van Zee et al. 1997; van den Hoek et al. 2000).

- LSBs are embedded in dark matter halos which are of lower density and more extended than the haloes around HSB galaxies, and they appear to be strongly dominated by Dark Matter at all radii (e.g., de Blok et al. 1996, 2001; McGaugh et al. 2001)

The star formation history of LSBs has been the subject of recent debate. The LSBs best studied in the optical and in the near-infrared are blue (e.g., Bergvall et al. 1999), indicating a young mean stellar age and/or metallicity. Morphologically, most studied LSBs have discs, but little spiral structure. The current massive star formation rates in LSBs are an order of magnitude lower than those of HSBs (van Zee et al. 1997); H I observations show that LSBs have high gas mass fractions, sometimes exceeding unity (Spitzak \& Schneider 1998;
McGaugh \& de Blok 1997). All these observations are consistent with a scenario in which LSBs are relatively unevolved, low mass surface density, low metallicity systems with roughly constant star formation rate. However, this scenario has some difficulty accommodating giant LSBs like Malin 1 (Bothun et al. 1987), see, e.g., Boissier et al. (2003).

This study of infrared LSBs was also intended to investigate the possibility of there being a substantial population of red LSBs like those reported in the optical $U B V R I$-band study of O'Neil et al. (1997). Follow-up H I line studies initially indicated that some of these red LSBs with rotational speeds exceeding $200 \mathrm{~km} \mathrm{~s}^{-1}$ did not seem to follow the "standard" Tully-Fisher relation established for HSB galaxies, in the sense that they appeared to be severely underluminous for their total mass (O'Neil et al. 2000). More recent H I imaging observations by Chung et al. (2002) indicate that the rotational properties and total H I mass of these red LSBs had been strongly overestimated due to confusion within the telescope beam with neighbouring galaxies. An infrared-selected sample should allow us to identify whether there is a significant population of very red LSBs.

In the present paper a description is given of the 2MASS LSB galaxy sample selection and its results, while 21-cm HI line observations from Arecibo and Nançay will be presented in Papers II and III, respectively (Monnier Ragaigne et al. 2003b,c), optical $B V R I$-band CCD surface photometry of a sub-sample of 35 2MASS LSB galaxies will be presented in Paper IV (Monnier Ragaigne et al. 2003d) and an analysis of the full data set will be presented in Paper V. Models of the evolution of the 2MASS galaxies presented in Paper IV and of other samples of LSB galaxies are presented in Boissier et al. (2003).

The 2MASS survey is described in Sect. 2, and the 2MASS LSB galaxies sample selection in Sect. 3. Basic photometric properties of the sample are presented in Sect. 4 and compared with those of others samples of LSB and HSB galaxies. Subsets of the sample observed by us at other wavelengths are described in Sect. 5.

\section{The 2MASS all-sky near-infrared survey}

The Two Micron All Sky Survey, 2MASS, has imaged the entire celestial sphere in the near-infrared $J(1.25 \mu \mathrm{m})$, $H(1.65 \mu \mathrm{m})$ and $K_{\mathrm{s}}(2.16 \mu \mathrm{m})$ bands using two identical dedicated 1.3-meter telescopes, designed specifically for the survey. One goal of the survey was to probe the nearby Universe in detail out to redshifts of $z \sim 0.1$. Accordingly, it is highly uniform in both photometric and astrometric measurements. The 2MASS Extended Source Catalog (XSC) consists of more than 1.6 million galaxies brighter than 14 th mag at $K_{\mathrm{s}}$ with angular diameters greater than $\sim 10^{\prime \prime}$. The photometry includes accurate Point Spread Function (PSF)-derived measures and a variety of circular and elliptical aperture measures, fully characterizing both point-like and extended objects. The position centroids have an astrometric accuracy better than $\sim 0 \prime$..5. In addition to tabular information, 2MASS archives full-resolution images for each extended object, enabling detailed comparison with other imaging surveys. 
Though 2MASS perforce needed to sacrifice depth for allsky coverage, and is therefore less deep than some of the dedicated optical imaging surveys made of LSB galaxies over limited areas of the sky, it should still be sensitive to the bright end of the LSB spectrum, particularly if there is a class of very red LSB objects. It has a 95\% completeness level in $J, H$ and $K_{\mathrm{S}}$ of 15.1, 14.3 and $13.5 \mathrm{mag}$, respectively, for "normal" galaxies (Jarrett et al. 2000); for LSB and blue objects the completeness limits are not yet known. In practice, 2MASS detects galaxies with central surface brightness values ranging from 14-20 mag $\operatorname{arcsec}^{-2}$ of which the LSB fraction with $18<\mu_{K 5}<20 \mathrm{mag} \operatorname{arcsec}^{-2}$ varies from $\sim 10 \%$ of the total 2MASS sample for large galaxies $\left(r_{K_{20}} \geq 20^{\prime \prime}\right)$ to $\sim 40 \%$ for the smaller galaxies $\left(20^{\prime \prime} \geq r_{K_{20}} \geq 10^{\prime \prime}\right)$. These near-infrared data will be less susceptible than optical surveys to the effects of extinction due to dust, both Galactic and internal to the galaxies. Also, the luminous mass distribution of galaxies is dominated by the older stellar populations, which emit most of their light in the near-infrared.

Initial results for galaxies detected by 2MASS, including LSB objects, are described in several publications (Schneider et al. 1997; Jarrett et al. 2000a,b; Hurt et al. 2000).

\subsection{The extended source processor}

The last major subsystem to run in the 2MASS quasi-linear data reduction "pipeline" is the extended source processor, GALWORKS. The primary role of the processor is to characterize each detected source and decide which sources are extended or resolved with respect to the point spread function (PSF). Sources that are deemed extended are measured further and the information is written into a separate table. In addition to tabulated source information, a small "postage stamp" image is extracted for each extended source from the corresponding $J, H$ and $K_{\mathrm{s}}$ Atlas images. The source lists and image data are stored in the 2MASS extended source database. By the time GALWORKS is run in the 2MASS pipeline, point sources have been fully measured with refined positions and photometry, band-merged, coordinate positions calibrated, Atlas images constructed, and the time-dependent PSF characterized for every Atlas image. The high-level steps that encompass GALWORKS include: (1) bright star (and their associated features) masking, (2) Atlas image background subtraction, (3) measurement of the stellar number density and confusion noise, (4) source parameterisation and attribute measurements, (5) star-galaxy discrimination, (6) refined photometric measurements, and finally (7) source and image extraction (see Jarrett et al. 2000a for more details).

The sample selected for this study was extracted before the 2MASS data acquisition was completed, using an earlier version of the software extraction algorithms. However, comparisons between the final data and the earlier results show they are consistent. We also used the full 2MASS database, not just the high signal-to-noise sources that form the final catalog, so that our sample includes many fainter sources than are part of the publicly released catalogs.

\subsection{Low Central Surface Brightness (LCSB) source processor}

There are some galaxies whose central surface brightness is too low to be detected by the standard 2MASS procedure, but whose total integrated flux is significant. These may include low surface brightness galaxies and dwarf galaxies. We will refer to these sources with the generic moniker: Low Central Surface Brightness (LCSB) galaxies. LCSB galaxies present a different challenge to GALWORKS than the typical "normal" galaxy 2MASS encounters. They are generally very faint (as measured in a standard aperture for "normal" galaxies) and they do not have well defined cores; see Jarrett (1998) for examples. The LCSB processor was an experimental algorithm executed last in the chain of operations that comprise GALWORKS. Sources detected by this algorithm did not meet the stringent reliability criteria for inclusion in the public 2MASS catalogue, but it did occasionally identify interesting LSB galaxies, as confirmed with deep optical images. There are also some examples of galaxies observed to be low surface brightness in the near-infrared that have normal surface brightness in the optical, see, e.g., Galaz et al. (2002).

The input to the LCSB processor is a fully cleaned Atlas image in each band, where stars and previously found extended sources have been entirely masked. The image is then blocked up (using three independent kernel sizes: $2 \times 2,4 \times 4$ and $8 \times 8$ pixels) and "boxcar" smoothed to increase the signal-tonoise ratio for large (but faint) objects normally hidden in the pixel noise. The detection step consists of 3- $\sigma$ threshold isolation of local peaks in cleaned, smoothed images. Source detections are then parameterised, with the primary measurements being: signal-to-noise ratio of the peak pixel, radial extent, integrated signal-to-noise ratio, surface brightness, integrated flux, and signal-to-noise ratio measurements using a $J+H+K_{\mathrm{s}}$ combined "super-coadd" image. In principle, the "super-coadd" provides the best medium from which to find faint LSB galaxies, given the effective increase in the signal-to-noise ratio.

The LCSB processor could be falsely triggered by faint stars and the wings of the PSF around bright stars. Meteor streaks (and other transient phenomena) that were not fully cleaned from the Atlas images could also generate numerous false LCSB sources. It is important to note that LCSBdetected sources were nearly always fainter than the catalogue limit of the 2MASS survey, so their exclusion from the released catalogue does not significantly compromise its completeness. Further information and some early science results with 2MASS LCSB galaxies can be found in Jarrett et al. (2000a).

\section{2MASS LSB galaxies selection}

After a period of testing with various combinations of photometric parameters, we defined a modus operandi for the sample selection based on as few 2MASS parameters as possible, which allowed the definition of a sample with low infrared surface brightness values. The 2MASS central surface brightness limit of $\sim 20 \mathrm{mag} \mathrm{arcsec}^{-2}$ in $K_{\mathrm{s}}$ band is equivalent to a $B$ surface brightness of $\sim 24 \mathrm{mag} \operatorname{arcsec}^{-2}$ for a typical spiral galaxy 
colour. This corresponds to fairly "bright LSB" galaxies, easily detected in deep photographic and CCD optical limitedcoverage surveys. However, as we shall show later in this paper, our selection criteria yield a LSB sample with interesting complementary and contrasting properties compared to opticallyselected samples.

\subsection{Selection using the $K_{s}$-band central surface brightness}

In order to select infrared LSB galaxies, our key photometric 2MASS parameter is $\mu_{K 5}$, the mean $K_{\mathrm{s}}$-band central surface brightness within a circle of radius 5 arcsec. This aperture was selected because for the great majority of faint galaxies in the 2MASS catalogue, this small fixed circular aperture gives good signal-to-noise statistics while avoiding problems due to confusion and missing flux in the faint outer parts of galaxies. The circular 5" radius aperture also appears more reliable than the peak central surface brightness measured in a single pixel of the original $1.8 \mathrm{~s}$ integration.

We selected sources with $\mu_{K 5}>18.0 \mathrm{mag} \operatorname{arcsec}^{-2}$. The usefulness of this criterion for the selection of LSB galaxies has been shown in Jarrett et al. (1998) for selecting LSB galaxies in the Coma cluster and SA57 region.

\subsection{Selection using source size}

The size of the sources is also important for the sample selection:

- A fixed-diameter aperture gives the surface brightness over different physical areas within the galaxy, depending on its distance. For angularly small galaxies, the fixed aperture gives a surface brightness closer to the mean over the whole galaxy.

- The reliability of the catalogue is related to the size of the objects. For very small sources, confusion with stars increases significantly

- We planned also to obtain observation in the $21 \mathrm{~cm} \mathrm{H}$ I radio line, and flux levels tend to be correlated with angular size.

For these reasons, we selected two samples of galaxies based on $r_{K_{20}}$, the $K_{\mathrm{s}}$-band semi-major axis at an isophotal level of $20 \mathrm{mag} \operatorname{arcsec}^{-2}$. Our primary sample is of sources with $r_{K_{20}}>20^{\prime \prime}$ (hereafter referred to as the "Large" 2MASS LSBs sample), and a secondary sample with $20^{\prime \prime}>r_{K_{20}}>10^{\prime \prime}$ (hereafter referred to as the "Small" 2MASS LSBs sample).

\subsection{Selection using colour range}

Two effects make galaxies appear "redder" in the 1 to $2 \mu \mathrm{m}$ window (see Fig. 14, Sect. 5, in the Explanatory Supplement to the 2MASS Second Incremental Data):

- their light is dominated by older stellar populations

- their redshift tends to transfer additional stellar light into the $2 \mu \mathrm{m}$ windows, boosting the $K_{\mathrm{s}}$ band flux relative to the $J$ band flux (K-correction)
In general, the $\left(J-K_{\mathrm{s}}\right)$ colour falls within a fairly narrow range for nearby galaxies, $0.8<\left(J-K_{\mathrm{s}}\right)<1.3$, and GALWORKS uses this fact to help discriminate galaxies from other Galactic extended sources. Because we are exploring a potentially unusual class of galaxies, we examined a wider colour range of sources from the "Working Survey Database" of 2MASS (including uncertain sources that do not meet the completeness and reliability criteria for the 2MASS catalogue). To exclude most artifacts and confusing sources, we select sources with $0.5<\left(J-K_{\mathrm{s}}\right)<2.0$. This range also allows for larger photometric uncertainties that are more likely to be found for LSB galaxies.

\subsection{Sky coverage}

The presently available 2MASS database covers all the sky. At the time when the sample was selected (end of 1999) the declination range of $\sim 0^{\circ}$ to $+12^{\circ}$ had not yet been covered, however, as can been seen in Fig. 1, which shows the sky coverage of the objects from our sample observed in the $\mathrm{H}$ I line.

To select our sample of LSB galaxies, we exclude the region inside the Zone of Avoidance $\left(|b|<10^{\circ}\right)$ where the confusion rate between multiple stars and galaxies is high, as well as the declinations not suited for observations with the Nançay and Arecibo radio telescopes.

Thus the area of the sky covered by our survey ranges from $-39^{\circ}$ to $\sim 0^{\circ}$ and from $\sim 12^{\circ}$ to $+60^{\circ}$ in declination, and the galactic latitude of our sources is $|b|>10^{\circ}$.

\subsection{Final sample}

Using the first and second algorithms described in Sects. 2.1 and 2.2, we selected, respectively, 34000 and 318 LCSB sources with a mean central surface brightness $\mu_{K 5}$ fainter than $18 \mathrm{mag} \operatorname{arcsec}^{-2}$. All sources have a radius $r_{K_{20}}$ larger than 10 arcsec.

In order to decide which of these faint sources really are galaxies, additional data on them were used that were listed in online databases such as NED (NASA Extragalactic Database) [http://nedwww . ipac . caltech.edu], LEDA (Lyon-Meudon Extragalactic Database) - recently incorporated in HyperLeda [http://www-obs.univ-lyon1/hypercat/] - and Aladin of the Centre de Données astronomiques de Strasbourg (CDS) [http://aladin.u-strasbg.fr], and by inspecting their Digital Sky Survey (DSS) optical images. As the acquisition and interpretation of these supplementary data for 34000 sources was deemed to be too laborious, and as we intended, after a first inspection of the sample, to concentrate our multi-wavelength observations (see Sect. 5) on the largersized objects, we limited this phase of the selection procedure to all 2000 objects in the subset of "large" sources $\left(r_{K_{20}}>20^{\prime \prime}\right)$ and to 3000 randomly selected objects in the subset of "small" sources $\left(20^{\prime \prime}>r_{K_{20}}>10^{\prime \prime}\right)$. Using this procedure, we selected a total of 3736 and 59 candidate 2MASS LSB galaxies from the sources extracted by the GALWORKS standard and LCSB algorithms, respectively. The total sample of 3796 objects constitutes our working database of 2MASS LSB galaxies. 

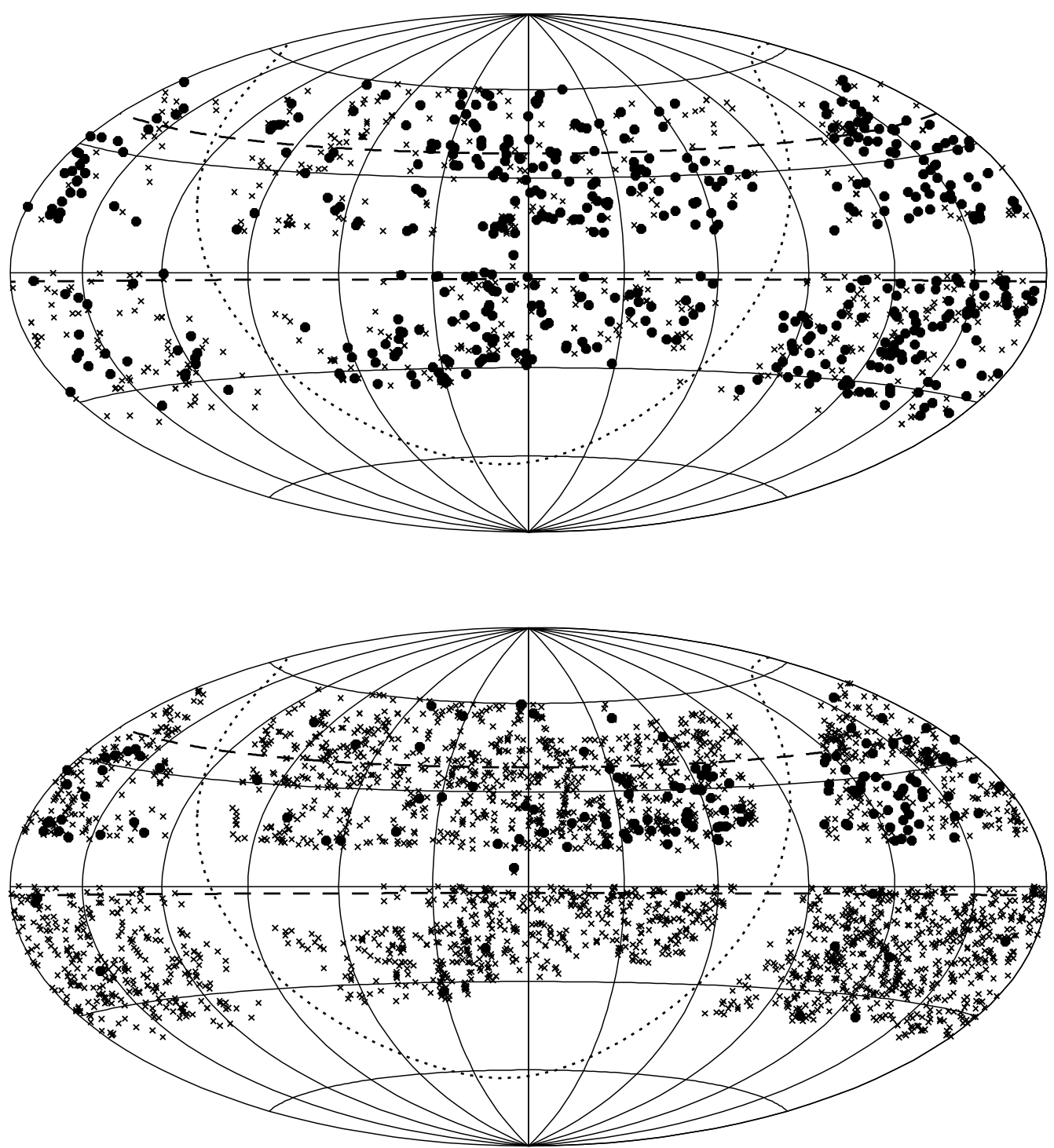

Fig. 1. The sky coverage of the selected sample of 2MASS LSB galaxies is illustrated by the distribution of the objects which were observed in H I. The plot is in equatorial coordinates; the dotted line indicates the Galactic plane and the dashed lines the $-2^{\circ}$ to $38^{\circ}$ declination range covered by the Arecibo radio telescope. All sources have declinations between $-39^{\circ}$ and $+60^{\circ}$. The upper panel is for the Large sources $\left(r_{K_{20}}>20^{\prime \prime}\right)$, the lower for the Small sources $\left(20^{\prime \prime}>r_{K_{20}}>10^{\prime \prime}\right)$. Dots indicate galaxies detected in H I, crosses undetected objects.

As the selected LCSB sources are not part of the standard 2MASS data released for public use, unlike the other sources, we show their near-infrared images in Fig. 2.

\section{Basic properties of the sample}

Some basic parameters of our working database of 3795 2MASS LSB galaxies are listed in Tables 1-3. We have divided the subsets listed in the Tables according to two criteria: processor and size. Tables 1 and 2 list the objects extracted using the standard algorithm for, respectively, the Large and Small sources, while Table 3 lists the Faint objects extracted using the LCSB algorithm.

It should be noted that the selection of the infrared sources presented in this work, which are all derived from 2MASS $J, H$ and $K_{\mathrm{s}}$-band imaging, was made in late 1999 , when work on the 2MASS database was still in full progress. The fiveyear evolution of both detection and source characterization was driven by improvements in the pipeline reductions and the image calibration. These are realized through preliminary database holdings and public "sampler" data releases, incremental data releases and finally the All Sky data release that achieved its milestone in March of 2003. Accordingly, the current sample is a mixture of these data releases, which are described in the 2MASS Explanatory Supplement, see URL http://www.ipac. caltech.edu/2mass/releases/ allsky/doc/explsup.html.

The Large and Small sources put in parentheses in Tables 1 and 2, respectively, are those that are only found in the All Sky working databases, and not in the subsequent, more reliable, public data releases. Only 23 of the 836 Large sources we selected from the working databases do not occur in later data 
releases, as do 27 from the 2900 Small sources. Of these 50 non-confirmed sources, 41 have an entry in the PGC and 24 in other optical galaxy catalogues.

The situation is different for the 59 Faint sources, as these were selected for our survey using a dedicated LSB source detection routine (see Sect. 2.2), and none of them appeared in the working databases. Seventeen of them do appear in later public releases, however: see Table 3, where, for the sake of consistency, we have also put the names of the Faint sources that are not found in later public data releases in brackets.

The basic parameters listed in Tables 1-3 are as follows:

1. No: the "work name", used throughout the present series of papers, which consists of the type of the source (L: large; S: Small; F: LCSB (Faint source) processor) followed by its number and an indication of its inclusion in other catalogues (P: only in PGC (see Paturel et al. 1989, as updated continuously in LEDA), $\mathrm{O}$ : in other catalogue(s), $\mathrm{N}$ : not previously catalogued). Sources in parentheses did not appear in later public data releases (see above);

2. 2MASS: gives the J2000.0 right ascension and declination coordinates of the 2MASS source;

3. PGC: the PGC catalogue entry, if available;

4. Other: refers to entries in other galaxy catalogues;

5. $\mu_{K 5}$ : the mean central surface brightness (in mag $\operatorname{arcsec}^{-2}$ ) measured within a radius of 5 arcsec around the source's centre in the $K_{\mathrm{s}}$ band;

6. $\mu_{B_{25}}$ : the mean $B$-band surface brightness (in mag $\operatorname{arcsec}^{-2}$ ) within the $D_{25}$ blue isophotal diameter, at a level of $25 \mathrm{mag} \operatorname{arcsec}^{-2}$, as listed in LEDA;

7. Obs: information on available observations: A for our Arecibo H I detections and "a" for the non-detections (see Paper II), N for our Nançay H I detections and " $n$ " for the non-detections (see Paper III), O for other published H I detections (from LEDA, see Paper V), and $\mathrm{P}$ for our BVRI optical surface photometry data (see Paper IV).

\subsection{MASS and comparison samples}

To study the basic properties of our galaxy sample, we compared various subsets to existing samples of optically selected High and Low Surface Brightness galaxies. We compared nearinfrared and optical data from LEDA for the objects in the following samples which were detected in 2MASS:

1. 2MASS L: our "Large" LSB galaxies from the 2MASS survey catalogue with a $20 \mathrm{mag} \operatorname{arcsec}^{-2} K_{\mathrm{s}}$-band isophotal radius $r_{K_{20}} \geq 20^{\prime \prime}$ [836 objects];

2. 2MASS S: same for the "Small" objects, with $20 " \geq r_{K_{20}} \geq$ $10^{\prime \prime}$ [2900 objects];

3. 2MASS F: 2MASS sources with lower central surface brightness, extracted using the LCSB algorithm (see Sect. 2.2) [59 objects];

4. NGC L: "classical" HSB galaxies from the NGC, with $r_{K_{20}} \geq 20^{\prime \prime}$ [1973 objects];

5. NGC S: same, with $r_{K_{20}} \leq 20^{\prime \prime}$ [252 objects];

6. LSB opt.: optically selected LSB galaxies from Impey et al. (1996) [83 objects].
For the last sample of 693 optically selected LSB galaxies (derived from the inspection of UK Schmidt photographic plates) 83 objects were found in the 2MASS working survey database (i.e., 12\%). These LSBs found in the 2MASS database have a central surface brightness in $B$ (measured as described by Sprayberry et al. 1996) between 18.2 and $25.0{\text { mag } \operatorname{arcsec}^{-2}}^{-2}$ with a mean value around 21.6 mag $\operatorname{arcsec}^{-2}$ (i.e., the Freeman value for HSB spiral discs) compared to the other objects in the Impey et al. sample, which have a median value around $22.8 \mathrm{mag} \operatorname{arcsec}^{-2}$.

\subsection{Infrared surface brightness in the $K_{s}$ band}

In Fig. 3 we compare several different measures of the $K_{\mathrm{s}}$-band surface brightness for the subsets of the 2MASS LSB sample and the optically selected samples:

- Central $K_{\mathrm{s}}$-band surface brightness: $\mu_{K 5}$ is the primary selection criterion for inclusion in our 2MASS LSBs sample, the mean surface brightness measured within a circle of 5 " radius at the centre of each galaxy. Both the Large and Small 2MASS samples exhibit the highest counts at the $18 \mathrm{mag} \mathrm{arcsec}^{-2}$ cutoff, while the sources detected with the LCSB algorithm peak about $1 \mathrm{mag} \operatorname{arcsec}^{-2}$ lower. The 2MASS-selected sources span the lower surface brightness range of the optically selected LSBs detected by 2MASS. By contrast, the NGC sample objects are, on average, about 1.5 to $2 \mathrm{mag} \mathrm{arcsec}^{-2}$ brighter than the Small and Large 2MASS samples, respectively. The difference between the two NGC samples appears to be due to the inclusion of more of the disc in the small galaxies within the $5^{\prime \prime}$ radius aperture.

- Peak $K_{\mathrm{s}}$-band surface brightness: $\mu_{0}$ is the highest surface brightness measured in any of the $2^{\prime \prime}$ camera pixels near the centre of the galaxy during the original observations. 2MASS made six observations in a dithered pattern so that one observation was likely to be well-centred on the galaxy nucleus and should normally have the highest surface brightness. The results for the various samples are approximately the same as for the central surface brightness, although the Large and Small 2MASS samples become better distinguished. The small galaxies have slightly higher peak surface brightness values on average because the $\sim 1^{\prime \prime}$ radius aperture is more dominated by the bulge than the $5^{\prime \prime}$ aperture, whereas the measurements of the larger galaxies were already more bulge dominated.

- Mean isophotal surface brightness and mean face-on isophotal surface brightness: $\mu_{K_{21}}$ and $\mu_{K_{21}}(i=0)$ are, respectively, the average surface brightness measured within the ellipse fit to the $K_{\mathrm{s}}$-band $21 \mathrm{mag} \operatorname{arcsec}^{-2}$ isophotal contour and this value corrected to face-on $\left(i=0^{\circ}\right)$. The latter value simply uses the major axis diameter as a measure of the face-on radius. These definitions corresponds to older, optical, definitions of surface brightness that used the $B=25$ mag $\operatorname{arcsec}^{-2}$ isophotal size, $D_{25}$. Note again that our 2 MASS samples exhibit some of the lowest infrared surface brightness values of any of the samples. As this figure shows, especially the Large sample is expected to contain a relatively many highly inclined, intrinsically quite LSB sources, due to selection effects (see Sect. 4.3). The optical LSB sample has relatively high surface brightness 

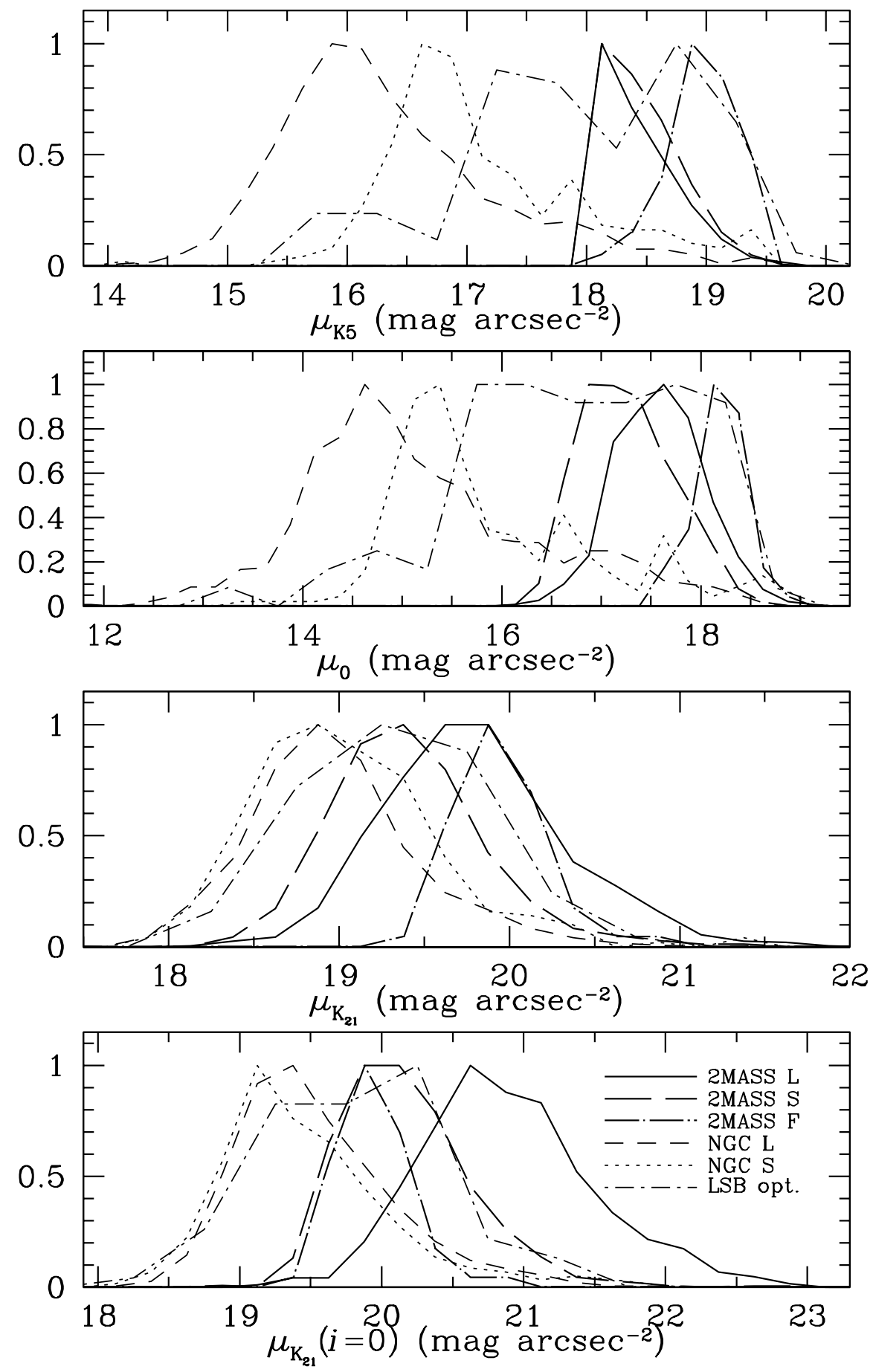

Fig. 3. Comparison between the normalized distributions of several measures of infrared surface brightness in the $K_{\mathrm{s}}$-band for the three subsamples of 2MASS LSB galaxies (Large/Small/Faint) with three other samples of galaxies, one of optically selected LSBs and two of HSB galaxies from the NGC, as described in Sect. 4.1. The plotted parameters, which are described and discussed further in Sect. 4.2, are: $\mu_{K 5}$ is the mean surface brightness measured within a circle of $5^{\prime \prime}$ radius at the centre of each galaxy, $\mu_{0}$ is the highest surface brightness measured at the centre of the galaxy, and $\mu_{K_{21}}$ and $\mu_{K_{21}}(i=0)$ are, respectively, the average surface brightness measured within the $21 \mathrm{mag}$ arcsec ${ }^{-2}$ isophotal contour in the $K_{\mathrm{s}}$-band and this value corrected to face-on $\left(i=0^{\circ}\right)$.

levels under this measurement scheme, because these galaxies may have a bright bulge even though their disc component has a low surface brightness, and a significant fraction may even have relatively bright discs, as the Impey et al. sample contains a significant number of non-LSB galaxies.

\subsection{Other infrared properties}

We compare a few other basic properties of the infrared emission in Fig. 4:

- $K_{\mathrm{S}}$-band isophotal radius: $r_{K_{20}}$ is the major-axis radius at the $K_{\mathrm{s}}=20 \mathrm{mag} \operatorname{arcsec}^{-2}$ elliptical isophote. Our 2MASS 

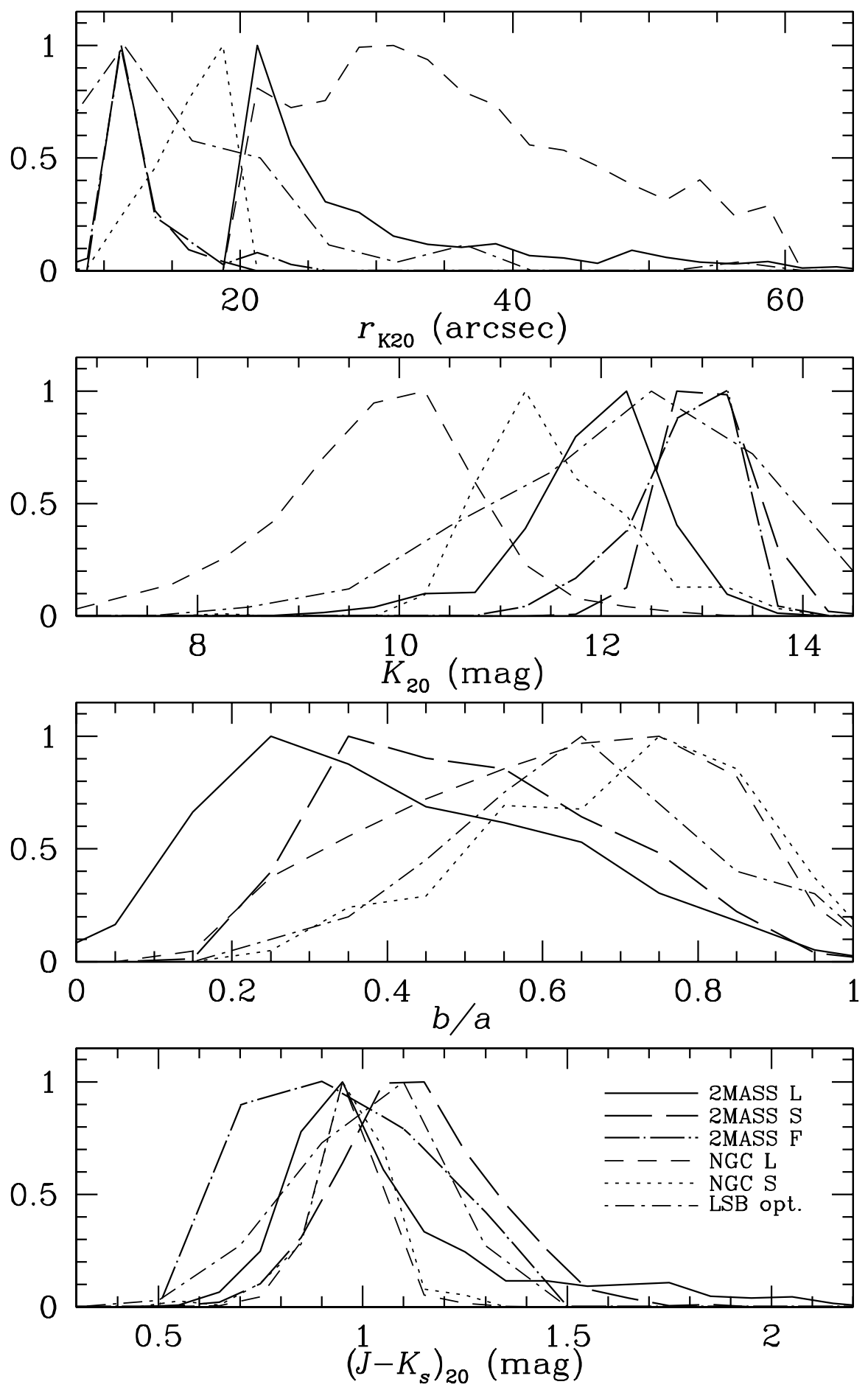

Fig. 4. As in Fig. 3, for a number of other infrared photometric parameters: $r_{K_{20}}$ is the major-axis radius at the 20 mag arcsec $^{-2}$ isophotal level in the $K_{\mathrm{s}}$ band, $K_{20}$ is the total magnitude within the $20 \mathrm{mag} \mathrm{arcsec}^{-2}$ elliptical isophote in the $K_{\mathrm{s}}$ band, $b / a$ is the minor-to-major axis ratio determined from an ellipse fit to the co-addition of the $J-, H$-, and $K_{\mathrm{s}}$-band images, and $(J-K)_{20}$ is the difference between the $J$-and $K_{\mathrm{s}}$-band magnitudes measured within the $K_{\mathrm{s}}=20 \mathrm{mag} \operatorname{arcsec}^{-2}$ elliptical isophote; note that the left half of the NGC S and NGC L curves overlap in the bottom panel. See Sect. 4.3 for further details on these parameters.

samples tend to be dominated by sources near the minimum radius, while the NGC samples are weighted toward angularly larger objects. The optical LSB sample is also dominated by smaller sources.

- $K_{\mathrm{s}}$-band isophotal magnitude: $K_{20}$ is the total magnitude within the $K_{\mathrm{s}}=20 \mathrm{mag} \operatorname{arcsec}^{-2}$ elliptical isophote.
Here again the 2MASS and optical LSB samples are weighted toward sources nearer the completeness limits of 2MASS, while the NGC samples include relatively bright objects.

- Infrared axis ratio: $b / a$ is determined from an ellipse fit to the co-addition of the $J$-, $H$-, and $K_{\mathrm{s}}$-band images. The fit is made at the $3-\sigma$ isophotal level relative to the background 
noise in each image. (The Faint 2MASS LCSBs sample was not measured in this way because of the low $\mathrm{S} / \mathrm{N}$ levels of the emission.) A sample of randomly oriented disc galaxies would exhibit a relatively flat distribution of $b / a$, while inclusion of elliptical galaxies and systems dominated by the bulge component tends to give a rising distribution toward larger values of $b / a$ (e.g., Mihalas \& Binney 1981). The NGC and optical LSB samples exhibit some tendency toward the distribution expected for bulge domination in the infrared, but the 2MASS samples show dominance by high-inclination systems, which is more pronounced for the Large sample than for the Small sources. This is due to the combined effects of the limits in observed central surface brightness and in apparent diameter, since, measured at a given isophotal level, highly inclined galaxies appear larger than more face-on objects with a similar intrinsic surface brightness profile. In particular the Large sample will contain an over-representation of highly inclined, intrinsically quite low surface brightness sources.

- Infrared colour: $(J-K)_{20}$ is the difference in $J$ - and $K_{\mathrm{s}}$-band magnitudes measured within the $K_{\mathrm{s}}=20 \mathrm{mag} \mathrm{arcsec}^{-2}$ elliptical isophote. The samples peak near $(J-K)=1$, which is found quite generally for all galaxy types. The LCSB sample is bluer (0.9 mag), while the Small 2MASS sample is somewhat redder $(1.2 \mathrm{mag})$ than the average. Although these colour differences may partly reflect differences in the K-correction due to galaxy redshift (bluer for nearer, redder for more distant), these are estimated to be of the order of $0.1 \mathrm{mag}$ at most, however, and cannot explain the much larger observed differences.

\subsection{Optical-infrared colours}

In Fig. 5 we compare optical and optical-infrared colours of the 2MASS and optical samples. We show several combinations based on total corrected extrapolated magnitudes from 2MASS measured in the $K_{\mathrm{s}}$ band and from LEDA measured in the $U$, $B, V$ and $I$ bands.

The total corrected $K_{\mathrm{s}}$-band infrared magnitudes, $K_{\mathrm{T}}$, were derived by modelling the increase in flux with successively larger apertures and extrapolating to a total magnitude. No corrections were applied for inclination or extinction, as these are in practice negligible at this wavelength. The estimated uncertainty in the extrapolated $K_{\mathrm{T}}$ magnitudes is of the order of $0.15 \mathrm{mag}$ for $K_{\mathrm{T}} \sim 13 \mathrm{mag}$, a typical value for the sample.

The total corrected optical magnitudes from LEDA are mostly derived from isophotal magnitudes, with corrections for galactic extinction, inclination and extinction effects (see Paturel et al. 1997). As the extinction correction depends on the morphological type, only galaxies with a $B_{\mathrm{T}_{\mathrm{c}}}$ value and a morphological type listed in LEDA were considered. This limits the percentage of useable 2MASS LSB galaxies in the Large, Small and Faint categories to $63 \%, 15 \%$ and 5\%, respectively, and the optically selected LSBs to $60 \%$.

In comparisons between the $U, B, V$, and $I$ bands, both the Large and Small 2MASS samples tend to be bluer than the optical samples: the optical LSB sample ("LSB opt" in plot) is next bluest, and the NGC samples exhibit the reddest colours. (The LCSB Faint source sample had too few sources with LEDA optical magnitudes to show meaningful colour distributions in these plots.) It is well established that the average HSB galaxy tend to be redder than the average, non-dwarf LSB (e.g., O'Neil et al. 1997; Gerritsen \& de Blok 1999; Burkholder et al. 2001). The presence in the Impey et al. "optical LSB" sample of a significant fraction of galaxies with relatively high central surface brightness, which can in fact be considered as HSBs, may well explain the intermediairy colours found for this sample.

Regarding the $\left(B_{\mathrm{T}_{\mathrm{c}}}-K_{\mathrm{T}}\right)$ colour, the Large and Small NGC galaxies have an average of 3.6 and standard deviations of 1.3 and 0.3 , respectively, while the optical LSBs are slightly bluer, $3.2 \pm 0.8$. Of the 2MASS samples, that of the Faint objects is the reddest on average, $3.2 \pm 0.6$, while the Small sources are also fairly red, $3.0 \pm 1.1$, but the mean of the Large 2MASS sample is $2.7 \pm 0.9$. Thus the infrared LSBs are not necessarily optical LSBs, according to our working definition of $\mu_{B_{0}, \mathrm{~d}}>22 \mathrm{mag} \operatorname{arcsec}^{-2}$, as the adopted $K_{\mathrm{s}}$-band selection limit of 18 mag arcsec ${ }^{-2}$ corresponds to a mean blue value of $\sim 20.8 \mathrm{mag} \mathrm{arcsec}^{-2}$ for their mean colour, though this includes the (unknown) contribution from the bulges.

\section{Discussion and further observations}

Through a simple definition of infrared surface brightness based on the magnitude in a small fixed aperture, we have identified a sample of infrared LSB galaxies. This definition has been shown to work well at selecting LSB galaxies in the Coma cluster and SA57 region (Jarrett et al. 1998), and we find that our sample has low infrared surface brightness levels compared to optically-selected samples of HSBs and LSBs detected by 2MASS.

We had initially expected an infrared-selected sample of LSB galaxies to have redder colours than optical samples, but instead it appears that by requiring $\mu_{K 5}>18 \mathrm{mag} \mathrm{arcsec}^{-2}$, we may have succeeded primarily in eliminating galaxies with significant bulge contributions. This already suggests that there is not a significant red population of LSB galaxies, although we note that the Large and Small 2MASS samples span a wide range of colours, particularly as measured in $B_{\mathrm{T}_{\mathrm{c}}}-K_{\mathrm{T}}$.

To better understand the infrared LSB galaxies' properties we have carried out a multi-wavelength study of subsets of manageable size. These results will be described in subsequent papers (II-V). For observations in the $21-\mathrm{cm} \mathrm{H}$ I line we selected two subsets: one of 367 Large, Small and Faint 2MASS LSBs for observations at Arecibo and another of 334 Large 2MASS LSBs for observations at Nançay, taking into account the difference in sensitivity and sky coverage of the two telescopes. The Arecibo and Nançay data will be presented in, respectively, Papers II and III of the present series. We have obtained CCD surface photometry of 35 objects in the $B, V$, $R$ and $I$-bands using the 1.5-meter telescope at the San Pedro Martír Observatory in Mexico. These surface photometry data will be presented in Paper IV. Finally, in Paper V we will further analyse the infrared LSBs to better understand, e.g. their properties as a function of their colours, investigating whether there is a significant population of red LSB galaxies, and to see if these objects follow the "standard" Tully-Fisher relation of HSB galaxies. In a related paper (Boissier et al. 2003), 

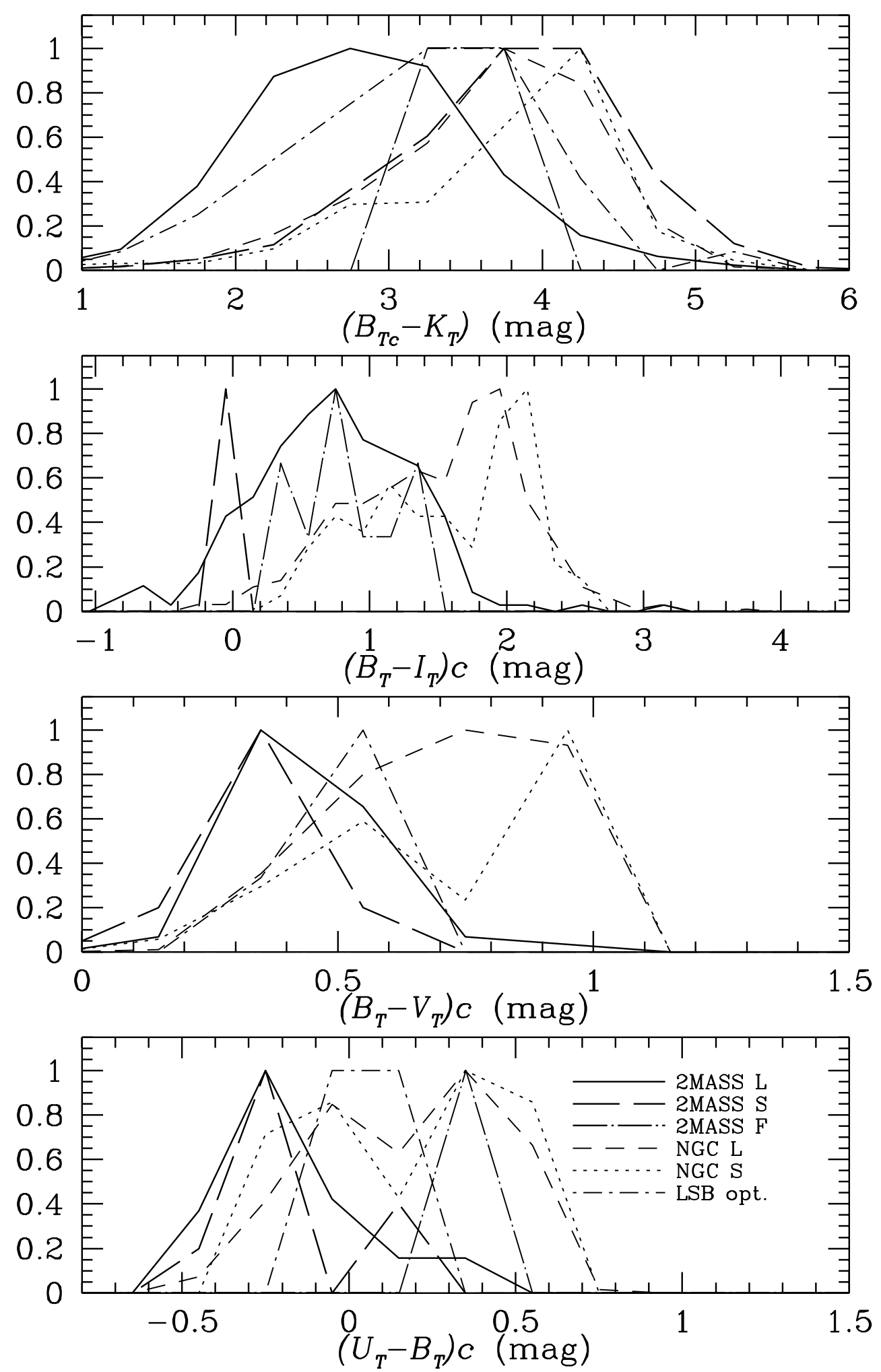

Fig. 5. As in Fig. 3 for a number of optical and optical-infrared colours, based on total corrected extrapolated magnitudes from 2MASS measured in the $K_{\mathrm{s}}$ band and from LEDA measured in the $U, B, V$ and $I$ bands. See Sect. 4.4 for a discussion of the plotted parameters.

we present models of the evolution of samples of LSBs, including the 2MASS objects for which we obtained optical surface photometry.

Acknowledgements. We want to thank the referee, Dr. N. Bergvall, for his comments. This publication makes use of data products from the Two Micron All Sky Survey, which is a joint project of the University of Massachusetts and the Infrared Processing and Analysis Center, funded by the National Aeronautics and Space Administration and the National Science Foundation. This research also has made use of the Lyon-Meudon Extragalactic Database (LEDA), recently 
incorporated in HyperLeda, the NASA/IPAC Extragalactic Database (NED) which is operated by the Jet Propulsion Laboratory, California Institute of Technology, under contract with the National Aeronautics and Space Administration and the Aladin database, operated at the CDS, Strasbourg, France. We acknowledge financial support from CNRS/NSF collaboration grant No. 10637.

\section{References}

Bergvall, N., Rönnback, J., Masegosa, J., \& Östlin, G. 1999, A\&A, 341, 697

Bell, E., Barnaby, D., Bower, R., et al. 2000, MNRAS, 312, 470

Boissier, N., Prantzos, N., Monnier Ragaigne, D., et al. 2003, MNRAS, in press [astro-ph/0304313]

Bothun, G. D., Impey, C. D., Malin, D. F., \& Mould, J. R. 1987, AJ, 94,23

Burkholder, V., Impey, C., \& Sprayberry, D. 2001, AJ, 122, 2318

Chung, A., van Gorkom, J. H., O’Neil, K., \& Bothun, G. D. 2002, AJ, 123,2387

de Blok, W. J. G., \& McGaugh, S. S. 1996, ApJ, 469, 89

de Blok, W. J. G., McGaugh, S. S., \& van der Hulst, J. M. 1996, MNRAS, 283, 18

de Blok, W. J. G., McGaugh, S. S., \& Rubin, V. C. 2001, AJ, 122, 2396

de Jong, R. S. 1996, A\&A, 313, 45

Disney, M. 1976, Nature, 263, 573

Freeman, K. C. 1970, ApJ, 160, 811

Galaz, G., Dalcanton, J. J., Infante, L., \& Treister, E. 2002, AJ, 124, 1360

Gerritsen J. P. E., \& de Blok, W. J. G. 1999, A\&A, 342, 655

Hurt, R. L., Jarrett, T. H., Kirkpatrick, J. D., et al. 2000, AJ, 20, 1876

Impey, C. D., Sprayberry, D., Irwin, M. J., \& Bothun, G. D. 1996, ApJS, 105, 209

Jarrett, T. 1998, Detection of Low Surface Brightness galaxies in 2MASS, in The Impact of Near-Infrared Sky Surveys on Galactic and Extragalactic Astronomy, ed. N. Epchtein (Dordrecht: Kluwer), 239

Jarrett, T. H., Chester, T., Cutri, R., et al. 2000a, AJ, 119, 2498
Jarrett, T. H., Chester, T., Cutri, R., et al. 2000b, AJ, 120, 298

Jarrett, T. H., Chester, T., Cutri, R., et al. 2003, AJ, accepted

Knezek, P., \& Wroten, J. 1994, BAAS, 185, No. 77.13

McGaugh, S. S., \& de Blok, W. J. G. 1997, ApJ, 481, 689

McGaugh, S. S., Rubin, V. C., \& de Blok, W. J. G. 2001, AJ, 122, 2381

Mihalas, D., \& Binney, J. 1981, in Galactic Astronomy: Structure and Kinematics (New York: Freeman), 338

Monnier Ragaigne, D., van Driel, W., O’Neil, K., et al. 2003a, A\&A, in press [astro-ph/0304553] (Paper II)

Monnier Ragaigne, D., van Driel, W., Schneider, S. E., Balkowski, C., \& Jarrett, T. H. 2003b, A\&A, in press [astro-ph/0305319] (Paper III)

Monnier Ragaigne, D., Papaderos, P., van Driel, W., et al. 2003c, A\&A, in preparation (Paper IV)

O’Neil, K., Bothun, G. D., Schombert, J., Cornell, M. E., \& Impey, C. D. $1997, \mathrm{AJ}, 114,2448$

O’Neil, K., \& Bothun, G. 2000, AJ, 529, 811

Paturel, G., Fouqué, P., Bottinelli, L., \& Gouguenheim, L. 1989, Monographies de la base de données extragalactiques, No. 1, vols. 1, 2 and 3 (Observatoires de Lyon et de Paris-Meudon)

Paturel, G., Botinelli, L., di Nella, H., et al. 1997, A\&AS, 124, 109

Schneider, S., Huchra, J., Jarrett, T., \& Chester, T. 1997, 2MASS Extragalactic Studies: Early Results from the Prototype Camer, in The Impact of Large-Scale Near-IR Sky Surveys, ed. F. Garzon, N. Epchtein, A. Omont, B. Burton, \& P. Persi (Dordrecht: Kluwer), 231

Spitzak, J. G., \& Schneider, S. E. 1998, ApJS, 119, 159

Sprayberry, D., Impey, C. D., \& Irwin, M. J. 1996, ApJ, 463, 535

van den Hoek, L. B., de Blok, W. J. G., van der Hulst, J. M., \& de Jong, T. 2000, A\&A, 357, 397

van der Hulst, J. M., Skillman, E. D., Smith, T. R., et al. 1993, AJ, 106,548

van Zee, L., Haynes, M. P., \& Salzer, J. J. 1997, Star Formation Activity in High M/L Galaxies, in Dwarf Galaxies: Probes for galaxy formation and Evolution, IAU GA Joint Discussion, Kyoto, Japan, 14 